\title{
Axial Energy Distribution in Disc-Shaped Tantalum and Aluminium Bremsstrahlung Conversion Targets
}

\author{
T.J. Williams* \\ AWE plc, Aldermaston, Reading, Berkshire, RG7 4PR, UK
}

\begin{abstract}
The axial distribution of energy deposited in bremsstrahlung conversion targets is modelled using the Monte Carlo $N$-Particle $\left(\mathrm{MCNP}^{\mathrm{TM}}\right)$ code. Systems comprising a disc-shaped target, segmented axially, and a point source of paraxial, monoenergetic electrons $(2,7,10$, or $14 \mathrm{MeV})$ are considered. Thick tantalum and aluminium targets and also tantalum targets of optimum thickness are modelled. Energy density, as a function of depth within the target, is found to depend on overall target thickness and to exhibit a maximum value at a particular depth. The far-field radiation polar diagram is calculated for X-rays exiting the target through its output face.
\end{abstract}

PACS numbers: 41.60.-m, 78.70.Ck, 02.70.Un

\section{Introduction}

Measured bremsstrahlung X-ray intensities, as a function of angle, are given in [1], [2] and [3] for electron source energies up to $\approx 3 \mathrm{MeV}$ for thick targets of various materials.

The work described here is a numerical investigation of the energy deposited by a pulse of electrons $(2,7,10$, or $14 \mathrm{MeV}$ ) incident on discs of tantalum (Ta, density $16.6 \mathrm{~g} \mathrm{~cm}^{-3}$ ) and aluminium (Al, density $2.7 \mathrm{~g} \mathrm{~cm}^{-3}$ ), respectively, used as target and blocking materials in radiographic machines at AWE and elsewhere. Tallying the bremsstrahlung photons, exiting the target through its output face, allows the associated radiation polar diagrams to be generated. The MCNP code [4] is used to simulate electron and photon transport and scattering processes in simple, disc-shaped target geometries with axial segmentation.

Such information could be used as an input to hydrocodes for hydrodynamic expansion studies since certain regions in a target material may change phase, or geometrically, if subjected to high energy densities. This could lead to plasma formation and alter the way an electron source couples with the target, which behaviour may be undesirable. A knowledge of energy deposition patterns within target materials is therefore important and systematic study could lead to enhanced target designs and increased on-axis radiographic dose.

\section{Bremsstrahlung X-ray production and effect of target thickness}

Bremsstrahlung photons are created when electrons interact with atomic nuclei in the target, a particularly

\footnotetext{
* e-mail: trevor.williams@awe.co.uk
}

efficient process in high- $Z$ (high atomic number) materials such as $\mathrm{Ta}(Z=73)$. The effect is less marked in $\mathrm{Al}(Z=13)$, which is principally used to absorb electrons that pass through the Ta conversion target while allowing most of the X-ray photons to be transmitted for radiographic purposes.

If a target is too thin, source electrons pass through it fairly readily, creating relatively few bremsstrahlung $\mathrm{X}$-ray photons. If the target is too thick, photons created in the first part of the target are attenuated by the remaining material. Thus, for a particular material, there is an optimum thickness which maximises on-axis dose from a conversion target. Interaction cross-sections are energy dependent.

\section{Outline of the MCNP models}

The target is disc shaped and divided into multiple segments along the axis of symmetry, usually with finer divisions towards the input face where there is a tendency for more energy to be absorbed. A paraxial (i.e. parallel to axis), monoenergetic point source of electrons is used with energies of $2,7,10$, or $14 \mathrm{MeV}$, directed along the axis towards the circular input face of the target. Targets of $\mathrm{Ta}(0.6 \mathrm{~cm}$ thick, $1 \mathrm{~cm}$ radius $)$ and $\mathrm{Al}(6 \mathrm{~cm}$ thick, $10 \mathrm{~cm}$ radius) are considered, as are Ta targets of radius $1 \mathrm{~cm}$ and optimum thickness that depends on source energy $(0.036 \mathrm{~cm}$ at $2 \mathrm{MeV}, 0.102 \mathrm{~cm}$ at $7 \mathrm{MeV}, 0.114 \mathrm{~cm}$ at $10 \mathrm{MeV}, 0.12 \mathrm{~cm}$ at $14 \mathrm{MeV})$.

A cell tally $\left({ }^{*} \mathrm{~F} 8\right)$ is used to determine the energy deposited in each segment, per source particle, by electrons and photons in combination. A surface tally ( $\left.{ }^{*} \mathrm{~F} 1\right)$, located at the output face of the target, is used to calculate the energies in cosine bins, per source particle, of bremsstrahlung X-ray photons exiting the target through its output face, allowing the radiation polar diagram to be calculated. 


\section{Analysis of MCNP output data}

The total energy deposited in each axial segment of the target, per source particle, is obtained from the ${ }^{*} \mathrm{~F} 8$ tally output, allowing the cumulative energy to be plotted straightforwardly as a function of depth within the target. The corresponding energy density is obtained by dividing the total energy deposited in a segment by the segment width, and is associated with the mid-point of the segment.

The radiation polar diagram can be calculated from the output of the cosine bins associated with the ${ }^{*} \mathrm{~F} 1$ tally, at the output face of the target, but care has to be taken when interpreting it. A cosine-bin limit is actually defined in terms of the cosine of the angle measured from the local normal to the surface. Though cosine bins can be used to plot the energy transported across a surface by photons (or electrons), as a function of angle, this should not be considered as defining a radiation polar diagram directly for two reasons. Firstly, particles can emanate from any point on the output surface of the target which has finite extent (i.e. it is not a point source). Secondly, since the geometry is three-dimensional (3D), particles can leave the surface at any azimuthal angle $\left(0-360^{\circ}\right)$ around an axis parallel to the local normal, for a given polar angle.

The first point can be neglected in the far field, since the small, planar, output surface of the target appears as a point source from distances sufficiently far, in which case polar angles can be considered measured from the axis of symmetry. The second point is accounted for by using solid angle, as detailed below.

The solid angle of a 3D surface, subtended at a point exterior to the surface, is defined as the area of the surface projected onto a unit sphere centred at the exterior point, there being $4 \pi$ steradian (sr) of solid angle for a complete spherical surface. For a band on a spherical surface, with angular limits $\alpha$ and $\beta$ from the axis of symmetry, the solid angle is given by: $\Omega=2 \pi(\cos \alpha-\cos \beta)$.

Thus, if angular limits $\alpha$ and $\beta$ define a cosine bin in MCNP, the angular energy density (in $\mathrm{MeV} / \mathrm{sr} / \mathrm{sp}$ where sp represents source particle) can be obtained by dividing the bin energy (in $\mathrm{MeV} / \mathrm{sp}$ ) by the solid angle given in the above expression. If this procedure is not adopted, the radiation polar diagram appears to have a maximum value off axis, as will be seen later.

\section{Results and discussion}

Graphs of energy density, and also cumulative energy deposited, as a function of target depth, are shown in Fig. 1a for a Ta target of thickness $0.6 \mathrm{~cm}$ at source energies of 2, 7, 10 and $14 \mathrm{MeV}$. Figures $1 \mathrm{~b}$ and $2 \mathrm{a}$ show respectively similar sets of curves for Ta targets of optimum thickness and an Al target of thickness $6 \mathrm{~cm}$.

The main features are as follows. For each level of source energy, a peak in the energy density occurs at a particular target depth. As source energy is increased,
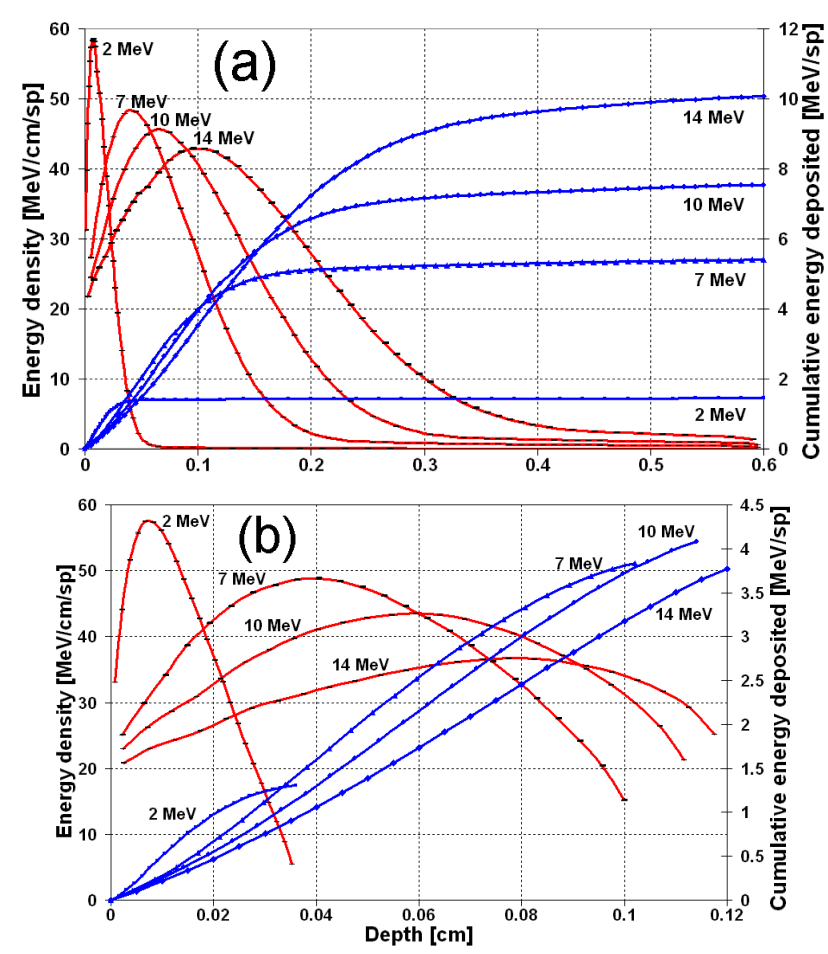

Fig. 1. (a) Energy deposited in a $0.6 \mathrm{~cm}$ thick Ta target by electrons and photons $(2,7,10$, and $14 \mathrm{MeV})$. (b) Energy deposited in optimum-thickness Ta targets by electrons and photons $(2,7,10$, and $14 \mathrm{MeV})$.

the height of the peak reduces whereas the target depth at which the peak occurs increases, as does the breadth of the peak. The energy density in Ta is found to be about 7 times that in $\mathrm{Al}$, which is close to the mass-density ratio of the materials.

In the case of Ta targets, for which both thick $(0.6 \mathrm{~cm})$ and optimum-thickness targets are considered, the peaks in the energy-deposition curves are similar in height and location, at source energies of 2 and $7 \mathrm{MeV}$. However, as source energy increases to 10 and $14 \mathrm{MeV}$, the peaks reduce in height and occur at a smaller depth for the optimum-thickness target, in relation to the thick target. This shows that energy deposition, as a function of target depth, depends on the overall thickness of target material. Essentially, this is because energy deposited at a particular depth within a target results from particles being transported back towards the source as well as those being transported away from it.

For targets of a given width $(0.6 \mathrm{~cm}$ Ta in Fig. 1a and $6 \mathrm{~cm} \mathrm{Al}$ in Fig. 2a), the total energy deposited is found to increase, as source energy is increased, which is most easily seen from the cumulative energy curves in the figures. The total energy absorbed by the target is always less than that delivered by the source electrons, but this is expected as the aim is to produce X-ray photons from the output face.

Figure $2 \mathrm{~b}$ shows plots of radiation polar diagrams, at source energies of $2,7,10$, and $14 \mathrm{MeV}$, for 

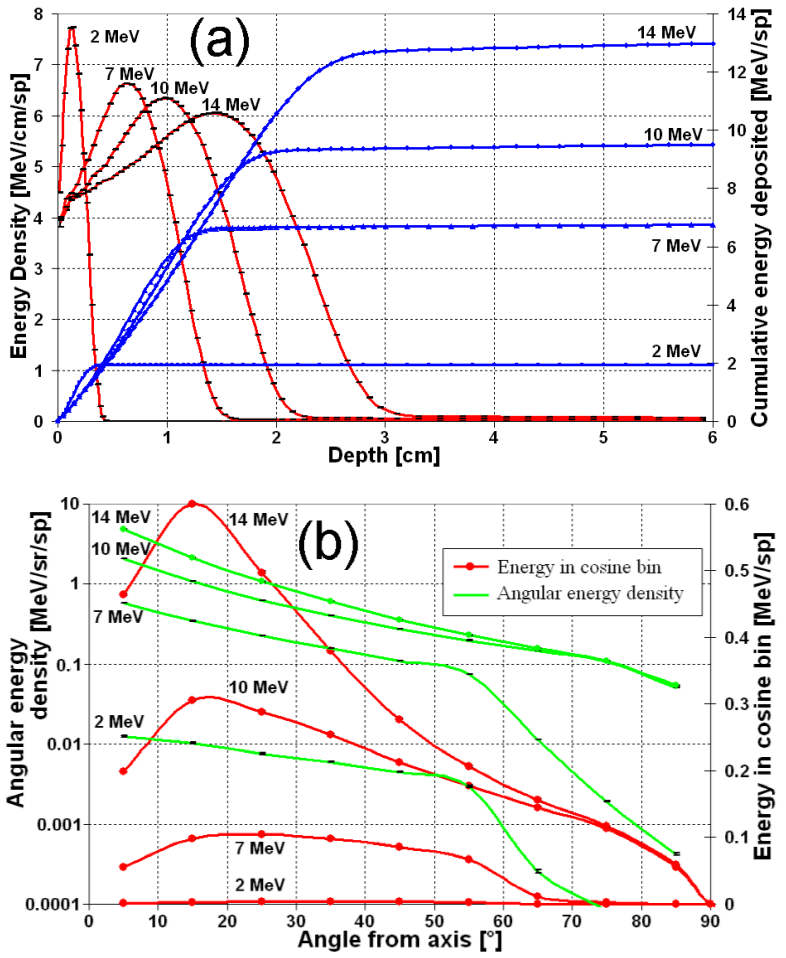

Fig. 2. (a) Energy deposited in a $6 \mathrm{~cm}$ thick $\mathrm{Al}$ target by electrons and photons $(2,7,10$, and $14 \mathrm{MeV})$. (b) Radiation polar diagrams and energies in cosine bins for bremsstrahlung photons exiting the output faces of optimum-thickness Ta targets $(2,7,10$, and $14 \mathrm{MeV})$.

bremsstrahlung photons exiting the output faces of Ta targets of optimum thickness. The curves with peaks are plots of the energies in the cosine bins of the ${ }^{*} \mathrm{~F} 1$ tally located at the output face of the target. They are not true radiation polar diagrams since no adjustment has been made to account for solid angle. However, if the values are adjusted for solid angle, as described in Sect. 4, curves result which rise monotonically as angle reduces (as in Fig. 2b).

If on-axis angular energy density is plotted as a function of source energy, a power law relationship is found to hold, the power being 2.60. Although the tally measure- ment is made at the output face of the target, rather than further away, this value is consistent with the accepted AWE value of 2.65 , for the power to which voltage must be raised, in the relationship between dose and machine output voltage.

\section{Further work}

It is planned to develop the MCNP model by dividing each axial segment of the target into a central disc and outer concentric rings of increasing diameter. This will allow energy deposition to be investigated radially as well as axially. Localised energy deposition data, output from the improved MCNP model, will be used as input to a hydrocode for hydrodynamic expansion studies. It is also intended to use the localised energy-deposition values, in conjunction with the thermal properties of target materials, to estimate temperature rise and the likelihood of phase change.

Further, it is proposed to model the electron source more realistically by considering beams of non-zero diameter or non-paraxial beams (e.g. with conical envelope), and beams with a distribution of energy (rather than monoenergetic).

\section{Acknowledgments}

(c) British Crown Copyright 2009/MOD.

\section{References}

[1] W.W. Buechner, R.J. Van de Graaff, E.A. Burrill, A. Sperduto, Phys. Rev. 74, 1348 (1948).

[2] W. Miller, J.W. Motz, C. Cialella, Phys. Rev. 96, 1348 (1954).

[3] D.H. Rester, W.E. Dance, J.H. Derrickson, J. Appl. Phys. 41, 2682 (1970).

[4] X-5 Monte Carlo Team, A General Monte Carlo N-Particle Transport Code, Version 5, Los Alamos National Laboratory, LA-UR-03-1987, 2003. 